\title{
Text messages to support e-cigarette use for smoking cessation: a tool for researchers
}

Sharon Cox ${ }^{1}$, Felix Naughton ${ }^{2}$, Catherine Kimber ${ }^{3}$, Vassilis Sideropoulos ${ }^{1}$, Eleni Vangeli ${ }^{3}$, Caitlin Notley ${ }^{2}$, Jamie Brown ${ }^{1}$, Daniel Frings ${ }^{3}$, Lynne Dawkins ${ }^{3}$

1 University College London, University of London

2 University of East Anglia

3 London South Bank University

Funding: This work was supported by a research grant from the Medical Research Council's (MRC) Public Health Intervention Development Scheme. Ref: MR/T002352/1 SC and JB receive salary support from Cancer Research UK (C1417/A22962)

Potential competing interests: SC has provided expert consultancy to providers of UK life insurance and the pharmaceutical industry on matters relating to smoking cessation aids. FN has provided consultancy to a medical equipment company to develop a smartphone app promoting effective use of nicotine replacement therapy and receives grants from Cancer Research UK. JB reports receiving grants from Cancer Research UK during the conduct of the study and receiving unrestricted research funding from pharmaceutical companies who manufacture smoking cessation medications to study smoking cessation outside the submitted work. DF received funding from Allen Carr's Easyway Ltd (ISRCN number:ISRCTN23584477). The trial was conducted independently, the protocol and analysis plan were both pre-registered and the research team were contractually free to independently publish the results of the trial regardless of the study outcome. DF has no other conflicts to declare. LD has provided consultancy for the pharmaceutical industry relating to the development of smoking cessation products.

\section{Abstract}

E-cigarettes are now the most popular quit aid chosen by smokers in England. Mobile phone text messages have been shown to be a useful tool in facilitating smoking cessation attempts by providing behaviour change support. To date, no published examples of text messages exist specifically aimed at smokers attempting to quit with e-cigarettes (vaping). As part of a parent study, we led an online call to vapers asking what advice and information they would give, in the form of a text message, to smokers wishing to quit smoking by vaping. We received 102 initial suggestions. Alongside behaviour change and smoking cessation experts, we checked the messages for readability and scientific accuracy, removed inappropriate messages, refined and edited others, and added additional messages. Messages were also grouped by theme and relevance to the stage of quitting. 95 messages were taken forward for further evaluation by 376 smokers, ex-smokers and vapers. Here we present the full set of 95 messages along with a final set of 78 that were selected and ordered for our parent study by two members of the research team along with four vapers. We encourage engagement with this resource and further validation from researchers interested in smoking cessation with e-cigarettes.

\section{Definitions}

E-cigarette

Defined by Robert West

Dual use of electronic cigarettes and cigarettes

Defined by Sharon Cox

Background 
E-cigarettes are now the most frequently used aid by smokers making a quit attempt in England ${ }^{[1]}$ and there is growing evidence for their efficacy within clinical trials ${ }^{[2][3]}$. The most recent Cochrane review of the evidence reported with moderate certainty that when used in randomised studies, e-cigarettes are more efficacious than nicotine replacement therapies in helping smokers to quit ${ }^{[4]}$. The popularity of e-cigarettes along with their growing evidence for helping people quit smoking is encouraging, but to date, there is a lack of evidence on what are the important components of e-cigarette use that help people to quit and what can be done to support smokers to use them more often. This is important as complete switching is associated with more frequent use ${ }^{[5]}$, and complete switching is associated with reduced harm ${ }^{[6]}$. Mobile phone text messaging has been shown to assist people across a range of health studies to change their behaviour and adapt to healthier habits ${ }^{[7]}$. Text message support is well used in smoking cessation studies and increases intervention effectiveness especially when compared to minimal support ${ }^{[8]}$. At the time of writing, there is limited evidence on their use within e-cigarette studies and no published examples of text messages specifically for new vapers. Here we present a set of text messages developed for a parent study ${ }^{[9]}$ designed with vapers, smokers and experts from within behavioural science.

An estimated one third current vapers also continue to smoke (known as dual use $)^{[10]}$, and many smokers have tried an ecigarette but have not continued use. There are several cited reasons for this:

Satisfaction: According to the cross sectional survey by Action on Smoking and Health $(\mathrm{ASH})^{[10]}$, of those who currently vape and do not smoke, two-thirds find vaping equally or more satisfying than smoking. However, for dual product users, around two thirds do not find vaping as satisfying. For those smokers who have tried an e-cigarette but discontinued use $80 \%$ report satisfaction as key reason for stopping vaping.

Perceptions of harm and safety concerns: Public perceptions of e-cigarettes are not aligned with the evidence. In the survey by $\mathrm{ASH}^{[10]}$, the proportion of smokers who correctly believe e-cigarette use is less harmful than smoking fell from $48 \%$ in 2019 to $39 \%$ in 2020 . Indeed in the same survey, $14 \%$ of smokers who had not yet tried an e-cigarette also cited concerns about product safety. Others have also shown that smokers perceptions of e-cigarettes became more negative since the highly publicised media reports of the outbreak of e-cigarette, or vaping, product use-associated lung injury (EVALI) ${ }^{[11]}$. Eventually, the CDC announced that the majority of cases were associated with inhalation of vitamin $\mathrm{E}$ acetate, an additive typically found in some tetrahydrocannabinol vaping devices obtained from informal sources.

Continued addiction: In each of the annual ASH surveys the most common reason given by current smokers for not trying an e-cigarette is feelings of swapping one addiction for another ${ }^{[10]}$.

Practical and technical problems: A number of published studies including the ASH survey have highlighted the difficulties people find with e-cigarettes ${ }^{[10]}$. In ethnographic work it has been cited that some individuals have been put off by the technical aspects of e-cigarettes and breakages lead to relapse to smoking ${ }^{[12][13]}$. Finding the right e-cigarette has also been cited as an important facilitator in being able to transition fully from smoking to exclusive vaping ${ }^{[12]}$. 
Public involvement can improve health research through the optimisation of specific research components which researchers may miss or undervalue ${ }^{[14]}$. Some of us have previously published on the importance of including those with lived experiences of quitting by vaping in advancing the science of tobacco harm reduction ${ }^{[15]}$. As shown above, there are several reasons why smokers may give up on vaping, therefore we wanted to include the experiences of vapers and smokers to develop a set of text messages which can be used to support smokers new to vaping to quit smoking. In January of 2019, we led an online (Twitter) call to vapers asking for help in developing text messages that could be used in a parent study, and that would be sent to smokers using an e-cigarette for first time during a quit attempt. We asked people to reflect on their own experiences of quitting and to consider what they would have found/would find useful in quitting smoking by vaping. We received 102 suggestions. These text messages were checked for readability and scientific accuracy. Messages that were factually incorrect, difficult to understand, very similar in content, inappropriate, or overly long and couldn't be shortened were removed. Others were reduced to fit the standard 160 characters for a text message or re-worded to improve readability. The research team also added their own messages and messages were themed and mapped onto behaviour change constructs of the COM-B model[ ${ }^{[16]}$. Table 1 (see attachments) presents the full set of 95 text messages that were evaluated in a survey to 376 smokers, ex-smokers and vapers in September 2019 (details to be published elsewhere). Some of these messages are similar in wording and researchers are encouraged to choose the messages which suits their population best rather than present them all. Messages used in our parent study (alongside other pre-validated generic smoking cessation text messages from cessation trials - not shown here) ${ }^{[17]}$ are indicated. Table 2 (see attachments) presents a reduced set of 78 messages, amended and ordered with the assistance of four vapers, which could be used as a standard set in a 3 month texting program. A methods paper describing the development of these messages is in preparation.

While not currently validated, these text messages fill an important gap in the published literature and provide examples of messages that can be used across a range of smoking cessation by e-cigarette studies. Importantly, these messages have been co-developed with people who have used, and are using, an e-cigarette, and that should also help researchers in the field as public involvement is increasingly more important in intervention design. We welcome feedback on these messages from the research community and further validation and input.

\section{References}

1. 'West, R., Beard, E, Kale, D., Brown, J.. (2020). Trends in electronic cigarette use in England.

2. `Peter Hajek, Anna Phillips-Waller, Dunja Przulj, Francesca Pesola, Katie Myers Smith, Natalie Bisal. (2019). $\underline{A}$ Randomized Trial of E-Cigarettes versus Nicotine-Replacement Therapy. N Engl J Med, vol. 380 (7), 629-637. doi:10.1056/nejmoa1808779.

3. 'Natalie Walker, Varsha Parag, Marjolein Verbiest, George Laking, Murray Laugesen, Christopher Bullen. (2020). 
Nicotine patches used in combination with e-cigarettes (with and without nicotine) for smoking cessation: a pragmatic. randomised trial. The Lancet Respiratory Medicine, vol. 8 (1), 54-64. doi:10.1016/s2213-2600(19)30269-3.

4. `Jamie Hartmann-Boyce, Hayden McRobbie, Nicola Lindson, Chris Bullen, Rachna Begh, Annika Theodoulou. (2020). Electronic cigarettes for smoking cessation. doi:10.1002/14651858.cd010216.pub4.

5. 'David T Levy, Zhe Yuan, Yuying Luo, David B Abrams. (2017). The Relationship of E-Cigarette Use to Cigarette Quit Attempts and Cessation: Insights From a Large, Nationally Representative U.S. Survey. doi:10.1093/ntr/ntx166.

6. 'Lion Shahab, Maciej L. Goniewicz, Benjamin C. Blount, Jamie Brown, Ann McNeill, K. Udeni Alwis. (2017). Nicotine. Carcinogen, and Toxin Exposure in Long-Term E-Cigarette and Nicotine Replacement Therapy Users. Ann Intern Med, vol. 166 (6), 390. doi:10.7326/m16-1107.

7. 'Katharine J. Head, Seth M. Noar, Nicholas T. lannarino, Nancy Grant Harrington. (2013). Efficacy of text messagingbased interventions for health promotion: A meta-analysis. Social Science \& Medicine, vol. 97, 41-48. doi:10.1016/j.socscimed.2013.08.003.

8. ^Robyn Whittaker, Hayden McRobbie, Chris Bullen, Anthony Rodgers, Yulong Gu, Rosie Dobson. (2019). Mobile phone text messaging and app-based interventions for smoking cessation. doi:10.1002/14651858.cd006611.pub5.

9. 'Catherine Kimber, Daniel Frings, Sharon Cox, Vassilis Sideropoulos, Felix Naughton, Jamie Brown. (2020). Tailored interventions to assist smokers to stop smoking using e-cigarettes (TASSE): Study protocol. Qeios. doi:10.32388/9rdlja.3.

10. ${ }^{a}, b, c, d, e$ Action on smoking and Health. (2020). Use of e-cigarettes-vapes among adults in Great Britain 2020.

11. 'Harry Tattan-Birch, Jamie Brown, Lion Shahab, Sarah E Jackson. (2020). Association of the US Outbreak of VapingAssociated Lung Injury With Perceived Harm of e-Cigarettes Compared With Cigarettes. JAMA Netw Open, vol. 3 (6), e206981. doi:10.1001/jamanetworkopen.2020.6981.

12. ${ }^{a}$ 'b Emma Ward, Sharon Cox, Lynne Dawkins, Sarah Jakes, Richard Holland, Caitlin Notley. (2018). A Qualitative Exploration of the Role of Vape Shop Environments in Supporting Smoking Abstinence. IJERPH, vol. 15 (2), 297. doi:10.3390/ijerph15020297.

13. ' Frances Thirlway. (2019). Nicotine addiction as a moral problem: Barriers to e-cigarette use for smoking cessation in two working-class areas in Northern England. Social Science \& Medicine, vol. $238,112498$. doi:10.1016/j.socscimed.2019.112498.

14. `Jo Brett, Sophie Staniszewska, Carole Mockford, Sandra Herron-Marx, John Hughes, Colin Tysall. (2012). Mapping the impact of patient and public involvement on health and social care research: a systematic review. Health Expect, vol. 17 (5), 637-650. doi:10.1111/j.1369-7625.2012.00795.x.

15. 'Caitlin Notley, Sharon Cox, Sarah Jakes, Louise Ross. (2019). What is the value of peer involvement in advancing tobacco harm reduction?. Harm Reduct J, vol. 16 (1). doi:10.1186/s12954-018-0275-1.

16. 'Susan Michie, Maartje M van Stralen, Robert West. (2011). The behaviour change wheel: A new method for characterising and designing behaviour change interventions. Implementation Sci, vol. 6 (1). doi:10.1186/1748-5908-642.

17. ' Felix Naughton, James Jamison, Sue Boase, Melanie Sloan, et al. (2014). Randomized controlled trial to assess the short-term effectiveness of tailored web-and text-based facilitation of smoking cessation in primary care ( $i Q$ uit in $P$ 
ractice). Addiction, vol. 109 (7), 1184-1193. doi:10.1111/add.12556. 